\title{
UNIFORM ASYMPTOTIC EXPANSIONS FOR SOLUTIONS OF THE PARABOLIC CYLINDER AND WEBER EQUATIONS
}

\author{
T. M. DUNSTER
}

Abstract. Asymptotic expansions are derived for solutions of the parabolic cylinder and Weber differential equations. In addition the inhomogeneous versions of the equations are considered, for the case of polynomial forcing terms. The expansions involve exponential, Airy and Scorer functions and slowly varying analytic coefficient functions involving simple coefficients. The approximations are uniformly valid for large values of the parameter and unbounded real and complex values of the argument. Explicit and readily computable error bounds are either furnished or available for all approximations.

Mathematics subject classification (2010): 33C10, 34E20, 34E05.

Keywords and phrases: Parabolic cylinder functions, turning point theory, WKB methods, asymptotic expansions.

\section{REFERENCES}

[1] M. Abu Zaytoon, T. Alderson And M. Hamdan, Weber's Inhomogeneous Differential Equation with Initial and Boundary Conditions, Int. J. Open Problems Compt. Math, 9 (2016), pp. 1-11, https://doi.org/10.12816/0033917.

[2] H. BuchHolz, The confluent hypergeometric function, Springer, Berlin, Heidelberg, 1969 , https : //doi .org/10.1007/978-3-642-88396-5.

[3] NIST Digital Library of Mathematical Functions, Release 1.0.25 of 2019-12-15, http://dlmf .nist.gov/, F. W. J. Olver, A. B. Olde Daalhuis, D. W. Lozier, B. I. Schneider, R. F. Boisvert, C. W. Clark, B. R. Miller, B. V. Saunders, H. S. Cohl, and M. A. McClain, eds.

[4] T. M. Dunster, Asymptotic solutions of inhomogeneous differential equations having a turning point, Stud. Appl. Math., 145 (2020), pp. 500-536, https ://doi .org/10.1111/sapm. 12326.

[5] T. M. Dunster, Liouville-Green expansions of exponential form, with an application to modified Bessel functions, Proc. Roy. Soc. Edinburgh Sec. A, 150 (2020), pp. 1289-1311, https://doi.org/10.1017/prm.2018.117.

[6] T. M. Dunster, A. Gil And J. Segura, Computation of asymptotic expansions of turning point problems via Cauchy's integral formula: Bessel functions, Constr. Approx., 46, (2017), pp. 645-675, http://dx.doi.org/10.1007/s00365-017-9372-8.

[7] T. M. Dunster, A. Gil And J. SEgurA, Uniform asymptotic expansions for Laguerre polynomials and related confluent hypergeometric functions, Adv. Comput. Math., 44 (2018), pp. 1441-1474, https : //doi.org/10.1007/s10444-018-9589-5.

[8] T. M. Dunster, A. Gil And J. Segura, Simplified error bounds for turning point expansions, Anal. Appl., (2020), https://doi.org/10.1142/S0219530520500104.

[9] A. Gil, J. SEgura AND N. M. TEMme, Integral representations for computing real parabolic cylinder functions, Numer. Math., 98 (2004), pp. 105-134, https://doi.org/10.1007/s00211-004-0517-x.

[10] A. Gil, J. Segura And N. M. Temme, Algorithm 850: Real parabolic cylinder functions $U(a, x)$, $V(a, x)$, ACM Trans. Math. Software, 32 (2006), pp. 102-112, https://doi.org/10.1145/1132973.1132978. 
[11] A. Gil, J. Segura And N. M. Temme, Computing the real parabolic cylinder functions $U(a, x)$, $V(a, x)$, ACM Trans. Math. Software, 32 (2006), pp. 70-101, https://doi.org/10.1145/1132973.1132977.

[12] A. Gil, J. Segura AND N. M. Temme, Algorithm 914: parabolic cylinder function $W(a, x)$ and its derivative, ACM Trans. Math. Software, 38 (2011), pp. Art. 6, 5, https://doi.org/10.1145/2049662.2049668.

[13] A. Gil, J. Segura And N. M. Temme, Fast and accurate computation of the Weber parabolic cylinder function $W(a, x)$, IMA J. Numer. Anal., 31 (2011), pp. 1194-1216, https://doi.org/10.1093/imanum/drq012.

[14] D. S. JonES, Parabolic cylinder functions of large order, J. Comput. Appl. Math., 190 (2006), pp. 453-469, https://doi.org/10.1016/j. cam.2005.04.009.

[15] D. A. Nield AND A. V. KuZnetsov, The effect of a transition layer between a fluid and a porous medium: shear flow in a channel, Transp. Porous Media, 78 (2009), pp. 477-487.

[16] F. W. J. OLver, Uniform asymptotic expansions for Weber parabolic cylinder functions of large orders, J. Res. Nat. Bur. Stand., 63B (1959), pp. 131-169.

[17] F. W. J. Olver, Second-order linear differential equations with two turning points, Philos. Trans. R. Soc. A, 278 (1975), pp. 137-174, https://doi.org/10.1098/rsta.1975.0023.

[18] F. W. J. OLver, Asymptotics and special functions, A K Peters Ltd., Wellesley, MA, 1997. Reprint of the 1974 original [Academic Press, New York].

[19] A. P. Prudnikov, I. U. A. Brychkov and O. I. Marichev, Integrals and Series: Special functions, Integrals and Series, Gordon and Breach Science Publishers, 1986.

[20] N. M. Temme, Numerical and asymptotic aspects of parabolic cylinder functions, J. Comput. Appl. Math., 121 (2000), pp. 221-246, https://doi .org/10 .1016/S0377-0427(00) 00347-2.

[21] N. M. Temme, Asymptotic methods for integrals, vol. 6 of Series in Analysis, World Scientific Publishing Co. Pte. Ltd., Hackensack, NJ, 2015. 